\title{
THE ONE-PHASE SUPERCOOLED STEFAN PROBLEM WITH A CONVECTIVE BOUNDARY CONDITION
}

\author{
$\mathrm{BY}$
}

DOMInGO A. TARZIA (Dpto. Matemática, Univ. Austral., Rosario, Argentina)

AND

CRISTINA V. TURNER (FaMAF, Univ. Nac. de Córdoba, Córdoba, Argentina)

\begin{abstract}
We consider the supercooled one-phase Stefan problem with convective boundary condition at the fixed face. We analyse the relation between the heat transfer coefficient and the possibility of continuing the solution for arbitrarily large time intervals.
\end{abstract}

I. Introduction. In this paper we study the following problem:

Problem I: Find $\theta(y, \tau)$ the temperature and $r(\tau)$ the free boundary such that $r(\tau)$ is Lipschitz continuous for $\tau>0$;

$\dot{r}(\tau)$ is continuous for $\tau>0$;

$\theta(y, \tau)$ is continuous for $\tau>0$ and $0 \leq y \leq r(\tau)$;

$\theta_{\tau}(y, \tau), \theta_{y y}(y, \tau)$ are continuous for $\tau>0$ and $0<y<r(\tau)$;

$\theta_{y}(y, \tau)$ is continuous for $\tau>0,0 \leq y \leq r(\tau)$;

$r(\tau)$ and $\theta(y, \tau)$ obey the conditions:

$$
\begin{gathered}
\theta_{\tau}=\alpha \theta_{y y}, \quad 0<y<r(\tau), \quad 0<\tau<\tau_{0} \\
\theta(r(\tau), \tau)=0, \quad 0<\tau<\tau_{0} \\
k \theta_{y}(r(\tau), \tau)=-\rho \lambda \dot{r}(\tau), \quad 0<\tau<\tau_{0} \\
k \theta_{y}(0, \tau)=h(\theta(0, \tau)-g(\tau)), \quad \tau>0 \\
\theta(y, 0)=\theta_{0}(y), \quad 0 \leq y \leq b \\
r(0)=b
\end{gathered}
$$

The parameters are

$\alpha=\frac{k}{\rho c}=$ material thermal diffusivity $\left(\mathrm{m}^{2} / \mathrm{s}\right) ;$

$k=$ material thermal conductivity $\left(K J / s^{0} \mathrm{Cm}\right)$;

$\rho=$ material density $\left(K g / \mathrm{m}^{3}\right)$;

$\lambda=$ latent heat of melting $(K J / K g)$;

Received December 27, 1993.

1991 Mathematics Subject Classification. Primary 35R35, 80A22.

Key words and phrases. Supercooled one-phase Stefan problem, phase-change problem, parabolic free boundary problem, oxygen-consumption problem. 
$h=$ fluid to material surface heat transfer coefficient $\left(\mathrm{KJ} / \mathrm{s}^{0} \mathrm{Cm}^{2}\right)$;

$g(\tau)=$ ambient fluid temperature $\left({ }^{\circ} C\right)$;

$\rho c=C=$ specific heat $\left(K J /{ }^{\circ} C K g\right)$.

The melting front at time $\tau$ is $r(\tau)$ while $\theta(y, \tau)$ is the temperature at position $y$ and time $\tau$.

It is known that a solution to Problem I exists [1], when $\theta_{0}(y) \leq 0$ and $g(\tau) \leq 0$. This problem is often referred to as a mathematical scheme for the freezing of a supercooled liquid (although this simple scheme for such a nonequilibrium phenomenon is far from being satisfactory) [3].

The freezing of a supercooled liquid is due to convective heat transfer from a fluid with ambient temperature $g(\tau)$ flowing across the face $x=0$. The adimensional problem is obtained by the following transforms:

$$
\begin{gathered}
x=\frac{y}{b}, \quad t=\frac{k \tau}{\rho c b^{2}}, \\
z(x, t)=\frac{c}{\lambda} \theta(y, \tau), \quad s(t)=\frac{r(\tau)}{b} .
\end{gathered}
$$

Then the variables $(T, s, z)$ satisfy the problem

Problem II:

(1.1) $z_{x x}=z_{t}$, in $D_{T}$;

(1.2) $s(0)=1$;

(1.3) $z(s(t), t)=0,0<t<T$;

(1.4) $z_{x}(s(t), t)=-\dot{s}(t), 0<t<T$;

(1.5) $z(x, 0)=\varphi(x), 0<x<1$;

(1.6) $z_{x}(0, t)=\beta[z(0, t)-G(t)], 0<t<T$,

where $\beta=\frac{h}{k b}$ is an adimensional parameter, and

$$
\begin{gathered}
D_{T}=\{(x, t) \mid 0<x<s(t), 0<t<T\}, \\
G(t)=\frac{c}{\lambda} g\left(\frac{b^{2} \rho c t}{k}\right) .
\end{gathered}
$$

II. The one-phase supercooled Stefan problem. In this section we consider the following hypotheses:

$$
\varphi(x) \leq 0,0<x<1 \text { and } G(t) \leq 0, t>0
$$

and the compatibility condition

$$
\varphi^{\prime}(0)=\beta[\varphi(0)-G(0)]
$$

The first simple properties of the solution of (1.1)-(1.6) are summarized in the following proposition: 
Proposition 2.1. If $(T, s, z)$ is a solution of Problem II, then

i) $z \leq 0$ in $D_{T}$.

ii) $\dot{s}(t)<0, t>0$.

iii) If $\dot{G}(t) \leq 0, \varphi(x) \geq G(0)=\max _{t>0} G(t)$, then $z \geq G(t)$ in $D_{T}$.

iv) If $\varphi^{\prime} \geq 0, \dot{G} \leq 0$, then $z_{x} \geq 0$ in $D_{T}$.

v) If $\dot{G} \geq 0, \varphi^{\prime \prime}>0$, then $z_{t}>0$ in $D_{t}$.

Proof. i), ii), and iv) follow from the maximum principle.

iii) follows from the minimum principle applied to $w=z-G$, where $w$ satisfies the following equation: $w_{x x}-w_{t}=\dot{G}$. Then the minimum of $w$ is on the boundary.

$\mathrm{v})$ follows from the maximum principle applied to $v=z_{t}=z_{x x}$.

Proposition 2.2. If $(T, s, z)$ satisfy (1.1)-(1.6) of Problem II, then the following integral representations are satisfied:

$$
\begin{gathered}
s(t)=1+\int_{0}^{1} \varphi(x) d x-\int_{0}^{t} z_{x}(0, \tau) d \tau-\int_{0}^{s(t)} z(x, t) d x, \\
\frac{s^{2}(t)}{2}=\frac{1}{2}+\int_{0}^{1} x \varphi(x) d x+\int_{0}^{t} z(0, \tau) d \tau-\int_{0}^{s(t)} x z(x, t) d x, \\
s(t)\left[1+\frac{\beta}{2} s(t)\right]=1+\frac{\beta}{2}+\int_{0}^{1}(1+\beta x) \varphi(x) d x+\int_{0}^{t} \beta G(\tau) d \tau \\
\frac{\beta s^{4}(t)}{24}+\frac{s^{3}(t)}{6}=\frac{\beta}{24}+\frac{1}{6}+\int_{0}^{1}\left(\frac{\beta x^{3}}{6}+\frac{x^{2}}{2}\right) \varphi(x) d x \\
-\int_{0}^{s(t)}\left(\frac{\beta x^{3}}{6}+\frac{x^{2}}{2}\right) z(x, t) d x+\iint_{D_{t}} z(x, t)(\beta x+1) d x d \tau .
\end{gathered}
$$

Proof. Consider Green's identity

$$
\iint_{D_{t}}\left(v L u-u L^{*} v\right) d x d \tau=\oint_{\partial D_{t}}\left(v u_{x}-u v_{x}\right) d \tau+u v d x
$$

where $L$ denotes the heat operator and $L^{*}$ its adjoint and the formulae $(2.1)-(2.2)$ are obtained by setting $u=z(x, t)$ and $v=1$ and $x$ respectively. (2.3) follows from (2.1) plus $\beta$ times (2.2), and (2.4) is obtained by using $v=\frac{\beta x^{3}}{6}+\frac{x^{2}}{2}$.

REMARK 1. In the following sections we denote

$$
Q(t)=1+\frac{\beta}{2}+\int_{0}^{1}(1+\beta x) \varphi(x) d x+\int_{0}^{t} \beta G(\tau) d \tau .
$$

If $\varphi(1)=0, \varphi(x)$ is Hölder continuous for $x=1$, and $G(t)$ is piecewise continuous on every interval $(0, t), t>0$, this problem possesses one solution for suitable $T$ "sufficiently small" (see [1], [2], [3] where uniqueness and continuous dependence are also discussed).

Moreover, if a solution exists, then three cases can occur (see [1], Theorem 8 and [2]).

(A) The problem has a solution with arbitrarily large $T$.

(B) There exists a constant $T_{B}>0$ such that $\lim _{t \rightarrow T_{B}} s(t)=0$.

(C) There exists a constant $T_{C}>0$ such that inf $t \in\left(0, T_{C}\right) s(t)>0$ and $\lim _{t \rightarrow T_{C}} \dot{s}(t)=$ $-\infty$. 
We shall investigate the occurrence of these cases in connection with the behavior of the initial data $\varphi$, the adimensional temperature $G$ of the external fluid, and the adimensional coefficient $\beta$.

Our next aim will be to look for some conditions on $\varphi, G$, and $\beta$ giving an a priori characterization of cases $(\mathrm{A}),(\mathrm{B})$, and $(\mathrm{C})$.

Proposition 2.3. If $\dot{G} \leq 0, \varphi(x) \geq G(0)$, and the solution $(T, s, z)$ of Problem II is case (B), then $Q\left(T_{B}\right)=0$.

Proof. Setting $t \rightarrow T_{B}$ in (2.3) and using the boundedness of $z$ obtained in Proposition 2.1 we conclude the thesis.

REMARK 2. $Q(t)$ is a decreasing function of time since $\dot{Q}(t)=\beta G(t)<0, \forall t$.

REMARK 3. If we consider the particular case where the initial temperature $\varphi(x)$ is zero and the temperature of the external fluid $G(t)=-B<0$ is a negative constant for all time, then $Q(t)$ is a linear function of time:

$$
Q(t)=1+\frac{\beta}{2}-t \beta B
$$

If the solution is case (B), then the stopping time is

$$
T_{B}=\frac{1+\frac{\beta}{2}}{\beta B}>0 \text {. }
$$

Proposition 2.4. If $(T, s, z)$ is a solution of Problem II, and the initial and boundary data satisfy the following hypotheses:

i) $\varphi(x) \geq M(x-1), 0 \leq x \leq 1,0<M<1$,

ii) $G(t) \geq-M$

and there exists a time $T_{B}$ such that $Q\left(T_{B}\right)=0$, then the solution $\left(T_{B}, s, z\right)$ is case $(B)$.

Proof. First we prove that $z(x, t) \geq M(x-1)$. This easily follows from the maximum principle applied to $w=z-M(x-1)$.

We replace this inequality in $(2.3)$ for $t=T_{B}$. Then $s\left(T_{B}\right)$ satisfies the following inequality:

$$
s\left(T_{B}\right)\left[(1-M)+s\left(T_{B}\right)\left[\frac{\beta(1-M)+M}{2}\right]+\beta s^{2}\left(T_{B}\right) \frac{M}{3}\right] \leq 0 .
$$

The quadratic form in brackets has coefficients $1-M>0$ and $\frac{\beta(1-M)+M}{2}>0$. Then $s\left(T_{B}\right)=0$.

Following [4] we obtain:

Proposition 2.5. Suppose that $t_{0}<T$ and $\lim _{t \rightarrow t_{0}} s(t)>0$, and suppose $\varphi$ satisfies the hypotheses iv) of Proposition 2.1. Moreover, $Q(t)>0$ for all $t \leq t_{0}$. Then if we define a function

$$
\eta(t)=\left\{\begin{array}{l}
\max \{x \in[0, s(t)] \mid z(x, t) \leq-1\} \\
0 \quad \text { if } z(x, t)>-1, x \in[0, s(t)]
\end{array}\right.
$$

it follows that

$$
\lim _{t \rightarrow t_{0}} \eta(t)<\lim _{t \rightarrow t_{0}} s(t) .
$$


Proof. Notice first that $\lim _{t \rightarrow t_{0}} s(t)$ exists because of Proposition 2.1. From iv) Proposition 2.1 we have $z(x, t) \leq-1$ in $[0, \eta(t)]$ and $-1<z(x, t) \leq 0$ in $(\eta(t), s(t)]$ for $t<t_{0}$. Let $\bar{s}=\lim \sup _{t \rightarrow t_{0}} \eta(t)$ and let $\left\{t_{n}\right\}$ be a sequence such that $t_{n} \rightarrow t_{0}$ and $\eta_{n}=\eta\left(t_{n}\right) \rightarrow \bar{s}$. Then from iii) of Proposition 2.2

$$
\begin{aligned}
s\left(t_{n}\right)\left(1+\frac{\beta}{2} s\left(t_{n}\right)\right) & =Q\left(t_{n}\right)-\int_{0}^{\eta\left(t_{n}\right)}(1+\beta x) z(x, t) d x-\int_{\eta\left(t_{n}\right)}^{s\left(t_{n}\right)}(1+\beta x) z(x, t) d x \\
& >Q\left(t_{n}\right)+\int_{0}^{\eta\left(t_{n}\right)}(1+\beta x) d x \\
& =Q\left(t_{n}\right)+\eta\left(t_{n}\right)+\frac{\beta}{2} \eta^{2}\left(t_{n}\right) .
\end{aligned}
$$

Performing the limit with $\eta \rightarrow \infty$,

$$
\begin{aligned}
\lim _{t \rightarrow t_{0}}\left(s(t)\left[1+\frac{\beta}{2} s(t)\right]\right) & \geq Q\left(t_{0}\right)+\lim _{t \rightarrow t_{0}}\left(\eta(t)\left(1+\frac{\beta}{2} \eta(t)\right)\right) \\
& >\lim _{t \rightarrow t_{0}}\left(\eta(t)\left[1+\frac{\beta}{2} \eta(t)\right]\right) .
\end{aligned}
$$

This above inequality is equivalent to

$$
\lim _{t \rightarrow t_{0}}\left[(s(t)-\eta(t))\left[1+\frac{\beta}{2}(s(t)+\eta(t))\right]\right]>0 .
$$

Since $1+\frac{\beta}{2}(s+\eta)>0, \forall t \leq t_{0}, \lim _{t \rightarrow t_{0}} s(t)-\eta(t)>0$.

Proposition 2.6. Let $(T, s, z)$ be a solution of Problem II such that $\varphi(x) \geq M(x-1)$, $0 \leq x \leq 1$, and $S_{T}=\inf _{t \in(0, T)} s(t)>0$. If there exist two constants $d \in\left(0, S_{T}\right)$, $z_{0} \in(0,1)$ such that $H d \leq z_{0}$, and

$$
z(s(t)-d, t) \geq-z_{0}, \quad 0 \leq t \leq T
$$

then

$$
\dot{s}(t) \geq \frac{\ln \left(1-z_{0}\right)}{d} .
$$

Proof. It is the same as the proof of Lemma 2.4 in [2]. (See also [4].)

Proposition 2.7. Let $(T, s, z)$ be a solution of Problem II and let $\varphi$ satisfy the hypotheses of Proposition $2.1 \mathrm{iv})$. Then if the solution is case $(\mathrm{C}), Q\left(T_{C}\right) \leq 0$.

Proof. Suppose $Q\left(T_{C}\right)>0$. Then from Proposition 2.5 the isotherm $z=-1$ is separated from the free-boundary. Using Proposition 2.6, $\dot{s}$ has a lower bound, which contradicts the case $(\mathrm{C})$.

Corollary 2.8. Let $(T, s, z)$ be a solution of Problem II and let $\varphi, G$ satisfy the following hypotheses:

i) $\varphi(x) \geq M(x-1), 0 \leq x \leq 1$;

ii) $G(t) \geq-M, 0<M<1$;

iii) $\dot{\varphi}(x) \geq 0,0 \leq x \leq 1$.

If the solution is case $(\mathrm{C})$, then $Q\left(T_{C}\right)<0$.

Proof. It follows from Propositions 2.4 and 2.7. 
Proposition 2.9. Let $(T, s, z)$ be a solution of Problem II, and let $\varphi$ and $G$ satisfy the following hypotheses:

i) $\varphi(x) \geq M(x-1), M>0,0 \leq x \leq 1$;

ii) $G \in L^{1}(0, \infty)$.

If the solution is case (A), then $Q(t) \geq 0, t>0$. Moreover, if $G(t) \geq-M(M>0)$, $\forall t>0$, then case (A) implies that $Q(t)>0, \forall t>0$.

Proof. We suppose that the thesis is not true. Then there exists a first time $T_{0}$ such that $Q\left(T_{0}\right)<0$. Since $Q(t)$ is a decreasing function, $Q(t)<0$ for $t>T_{0}$. We replace this estimation in the inequality (2.3) and we obtain the following inequality:

$$
-\int_{0}^{s(t)}(1+\beta x) z(x, t) d x=s(t)\left[1+\frac{\beta}{2} s(t)\right]-Q(t)>-Q\left(T_{0}\right), \quad t>T_{0} .
$$

Now we integrate the above equation with respect to time:

$$
\int_{t_{0}}^{t} \int_{0}^{s(t)}(1+\beta x) z(x, \tau) d x d \tau \leq Q\left(T_{0}\right)\left(t-T_{0}\right), \quad t \geq T_{0}
$$

then

$$
\iint_{D_{t}}(1+\beta x) z(x, t) d x d t \leq Q\left(T_{0}\right)\left(t-T_{0}\right), \quad t \geq T_{0} .
$$

The following step will be to seek an inequality that contradicts (2.6).

Using Eq. (2.4) we obtain

$$
\begin{aligned}
\iint_{D_{t}} z(x, t)(\beta x+1) d x d t= & -\int_{0}^{1}\left(\frac{\beta x^{3}}{6}+\frac{x^{2}}{2}\right) \varphi(x) d x+\frac{\beta s^{4}(t)}{24}+\frac{s^{3}(t)}{6} \\
& -\left(\frac{\beta}{24}+\frac{1}{6}\right)+\int_{0}^{s(t)}\left(\frac{\beta x^{3}}{6}+\frac{x^{2}}{2}\right) z(x, t) d x \\
\geq & -\left(\frac{\beta}{24}+\frac{1}{6}\right)+\int_{0}^{s(t)}\left(\frac{\beta x^{3}}{6}+\frac{x^{2}}{2}\right) z(x, t) d x
\end{aligned}
$$

From (2.3) and the hypotheses i) and ii)

$$
\begin{aligned}
\int_{0}^{s(t)}(1+\beta x) z(x, t) d x d t= & -s(t)\left[1+\frac{\beta}{2} s(t)\right]+\frac{\beta}{2}+\int_{0}^{1}(1+\beta x) \varphi(x) d x \\
& +\beta \int_{0}^{t} G(\tau) d \tau \\
\geq- & 1-\frac{\beta}{2}+1+\frac{\beta}{2}+M\left[\frac{1}{2}+\frac{\beta}{3}-\frac{\beta}{2}-1\right]-\beta\|G\|_{1 . t} \\
\geq & M\left[\frac{1}{2}+\frac{\beta}{6}\right]-\beta\|G\|_{1, t}=-C, \quad C>0, C \text { constant }
\end{aligned}
$$

where

$$
\|G\|_{1 . t}=-\int_{0}^{t} G(\tau) d \tau
$$


Since $0<x<s(t)<1$ and $z \leq 0$ in $D_{t}$ :

$$
\int_{0}^{s(t)} z(x, t)(1+\beta x) x^{2} d x>\int_{0}^{s(t)}(1+\beta x) z(x, t) d x \geq-C .
$$

Then

$$
\int_{0}^{s(t)}\left(\frac{\beta x^{3}}{6}+\frac{x^{2}}{2}\right) z(x, t) d x \geq-\frac{C}{2}-\frac{C}{6}=-D, \quad D>0 .
$$

We replace $(2.8)$ in $(2.7)$ :

$$
\iint_{D_{t}}(1+\beta x) z(x, t) d x d t \geq-\left(\frac{\beta}{24}+\frac{1}{6}\right)-D, \quad t>0
$$

This last inequality is in contradiction with (2.6). Then $Q(t) \geq 0, \forall t>0$. Moreover, if $G(t) \geq-M(M>0), \forall t>0$, the case (A) and Proposition 2.4 imply that $Q(t)>0$, $\forall t>0$.

\section{Asymptotic behavior of the solution.}

Proposition 3.1. Let $(T, s, z)$ be a solution of Problem II of case (A) under the hypotheses of Proposition 2.9 and (iii) of Proposition 2.1. Moreover, we assume that the limit of $G(t)$ when $t \rightarrow \infty$ exists. If we denote $Q_{\infty}=\lim _{t \rightarrow \infty} Q(t)$ and $s_{\infty}=\lim _{t \rightarrow \infty} s(t)$, then $s_{\propto}$ is given by

$$
s_{\infty}=\frac{-1+\sqrt{1+2 \beta Q_{\infty}}}{\beta} .
$$

Proof. The existence of the limit of $G(t)$ when $t \rightarrow \infty$ and $G \in L^{1}(0, \infty)$ assure that $\lim _{t \rightarrow \infty} G(t)=0$.

We denote by $z_{\infty}$ the limit of $z$ when $t$ tends to infinity. The existence of $\lim _{t \rightarrow \infty} z(x, t)$ is due to Proposition 2.1 and [6, Chapter 6]. The function $z_{\infty}$ satisfies: $z_{\infty}^{\prime \prime}=0$ in $\left(0, s_{\infty}\right)$, $z_{\infty}\left(s_{\infty}\right)=0, z_{\infty}^{\prime}(0)=\beta z_{\infty}(0)$; then $z_{\infty}(x)=0,0<x<s_{\infty}$.

Taking the limit when $t \rightarrow \infty$ in $(2.3)$, we have

$$
s_{\infty}\left[1+\beta \frac{s_{\infty}}{2}\right]-Q_{\infty}=0 .
$$

That means that $s_{\infty} \in(0,1)$ is the root of the above equation, that is, $(3.1)$.

Moreover, we have $s_{\infty}<1$ since

$$
s_{\infty}<1 \Leftrightarrow 1+2 \beta Q_{\infty}<(1+\beta)^{2} \Leftrightarrow 2 Q_{\infty}-2-\beta<0 .
$$

By taking the limit when $t \rightarrow \infty$ in (2.3) the last inequality always holds due to the following expression:

$$
2 Q_{\infty}-2-\beta=2 \int_{0}^{1}(1+\beta x) \varphi(x) d x-2 \beta\|G\|_{1}<0
$$

where $\|G\|_{1}=-\int_{0}^{\infty} G(\tau) d \tau$.

REMARK 4. We notice that

$$
s_{\infty}=0 \Leftrightarrow Q_{\infty}=0 \Leftrightarrow 1+\frac{\beta}{2}+\int_{0}^{1}(1+\beta x) \varphi(x) d x-\beta\|G\|_{1}=0 .
$$


Proposition 3.2. i) For any $t>0$ the free boundary of Problem II obeys the condition

$$
s(t) \geq 1+\int_{0}^{1} \varphi(x) d x+\beta t \inf _{0 \leq \tau \leq t} G(\tau) .
$$

ii) If $\dot{G} \leq 0$ and $\varphi(x) \geq G(0)$, then

$$
s^{2}(t) \geq 1+2 \int_{0}^{1} \varphi(x) d x+2 t \inf _{0 \leq \tau \leq t} G(\tau) .
$$

Proof. i) Using the integral representation (2.1) and i) of Proposition 2.1 we obtain (3.2). ii) It follows from the integral representation (2.2) and iii) of Proposition 2.1.

IV. The oxygen-consumption problem. As in [4] we are interested in the dependence on the temperature $G(t)$ of the external fluid at the fixed face $x=0$. If, in Problem II, we perform the classical transformation

$$
u(x, t)=\int_{x}^{s(t)}\left\{\int_{\hat{\imath}}^{s(t)}[1+z(\alpha, t)] d \alpha\right\} d \gamma
$$

then we obtain the following oxygen-consumption Problem III:

$$
\begin{aligned}
& u_{x x}-u_{t}=1, \quad \text { in } D_{t} \\
& s(0)=1 \\
& u(s(t), t)=u_{r}(s(t), t)=0, \quad t>0 \\
& u(x, 0)=H(x), \quad 0 \leq x \leq 1 ; \\
& u_{x}(0, t)-H^{\prime}(0)=\beta\left[u(0, t)-H(0)+\|G\|_{1 . t}\right], \quad t>0,
\end{aligned}
$$

where

$$
H(x)=\int_{x}^{1} \int_{\gamma}^{1}(1+\varphi(\alpha)) d \alpha d \gamma .
$$

From now on, in this section, we consider the following hypotheses for $\varphi$ :

$$
-1<\varphi(x) \leq 0, \quad 0 \leq x \leq 1 .
$$

Then

$$
H(x)>0,0 \leq x \leq 1 ; \quad H^{\prime}(x)<0,0 \leq x \leq 1 ; \quad H^{\prime \prime}(x)>0,0 \leq x \leq 1 .
$$

Proposition 4.1. Let $(T, s, u)$ be a solution of Problem III with $-1<\varphi \leq 0$ in $[0,1]$ and iii) of Proposition 2.1. Then $u(x, t)<H(x), x \in(0, s(t)), t>0$.

Proof. We apply the maximum principle to $W(x, t)=u(x, t)-H(x)$, which satisfies the following problem: $W_{x x}-W_{t}=1-H^{\prime \prime}(x)=-\varphi(x)>0, W(x, 0)=0, W(s(t), t)=$ $-H(s(t))<0$, and $W_{x}(0, t)=\beta\left[W(0, t)+\|G\|_{1 . t}\right]$.

We suppose there exists a $T_{0}>0$ such that $W\left(0, T_{0}\right)>0$. The point $\left(0, T_{0}\right)$ will be a maximum for $W$. Then the maximum principle implies $W_{x}\left(0, T_{0}\right)<0$, which is a contradiction to

$$
W_{x}\left(0, T_{0}\right)=\beta\left[W\left(0, T_{0}\right)+\|G\|_{1 . T_{0}}\right]>0 .
$$

We conclude that there does not exist such $T_{0}$. Then $W(x, t)=u(x, t)-H(x)<0$, $\forall x \in(0, s(t)), t>0$. 
Corollary 4.2. Let $(T, s, u)$ be a solution of Problem III. If $G(t)>-1, t>0$, then $u(x, t) \geq 0$ in $D_{t}$.

Proof. Using Proposition 2.1 iii) we obtain the following inequality:

$$
\begin{aligned}
u(x, t) & =\int_{x}^{s(t)} \int_{\gamma}^{s(t)} 1+z(x, t) d \alpha d \gamma \geq \int_{x}^{s(t)} \int_{\gamma}^{s(t)} 1+G(t) d \alpha d \gamma \\
& =(1+G(t))\left(\frac{s^{2}(t)}{2}-s(t) x+\frac{x^{2}}{2}\right) \geq 0 .
\end{aligned}
$$

We now consider some properties related to the qualitative behavior of the freeboundary.

Proposition 4.3. Let $(T, s, u)$ be a solution of Problem III. Then $s$ and $u$ satisfy the following integral representations:

i) $\int_{0}^{t} s(\tau) d \tau=\int_{0}^{1} H(x) d x-\int_{0}^{s(t)} u(x, t) d x-\int_{0}^{t}\left[\beta\left[u(0, \tau)-H(0)+\|G\|_{\tau}\right]+H^{\prime}(0)\right] d \tau$;

ii) $\int_{0}^{t} s^{2}(\tau) d \tau=\int_{0}^{1} x H(x) d x-\int_{0}^{s(t)} x u(x, t) d x+\int_{0}^{t} u(0, \tau) d \tau$;

iii) $\int_{0}^{t} s(\tau)[1+\beta s(\tau)] d \tau=\int_{0}^{1} H(x)[1+\beta x] d x-\int_{0}^{s(t)} u(x, t)[1+\beta x] d x$ $+\int_{0}^{t}\left[\|G\|_{\tau}+H^{\prime}(0)-H(0)\right] d \tau$.

Proof. i) and ii) follow by applying the Green's formula used in Proposition 2.2 and iii) is obtained as a combination of i) and ii).

We now address the question of how the solution to Problem III depends upon $G(t)$.

Proposition 4.4. The solution $(T, s, u)$ of Problem III depends monotonically on $G$. In particular, if $\left(T_{i}, s_{i}, u_{i}\right), i=1,2$, are the solutions for $G_{1}$ and $G_{2}$, respectively, and if $G_{1}(t)<G_{2}(t)$, then $s_{1}(t) \leq s_{2}(t)$ and $u_{1}(x, t) \leq u_{2}(x, t)$ however they are both defined.

Proof. This is seen by considering the difference

$$
v(x, t)=u_{2}(x, t)-u_{1}(x, t)
$$

at the points where they are both defined.

Let $t^{*}=\sup \left\{t>0 \mid u_{2}(0, t)>u_{1}(0, t)\right\}$ and let $t^{* *}=\sup \left\{t>0 \mid s_{2}(t)>s_{1}(t)\right\}$. Let us suppose that both $t^{*}$ and $t^{* *}$ are finite. By definition, $v$ satisfies the following problem:

$$
\begin{gathered}
v_{x x}=v_{t}, \quad x \in\left(0, s_{1}(t)\right), \quad t \in\left(0, t^{* *}\right) ; \\
v(x, 0)=0 ; \\
v\left(s_{1}(t), t\right)=u_{2}\left(s_{1}(t), t\right)>0 ; \\
v_{x}(0, t)=\beta\left[v(0, t)+\left(\left\|G_{2}\right\|_{1, t}-\left\|G_{1}\right\|_{1, t}\right)\right] .
\end{gathered}
$$

Claim 1: $t^{*} \neq t^{* *}$.

In order to prove that $t^{*}$ and $t^{* *}$ are different from each other, let us suppose that they are equal. Then

a) $s_{1}\left(t^{*}\right)=s_{2}\left(t^{*}\right)$

b) $\dot{s}_{1}\left(t^{*}\right)>\dot{s}_{2}\left(t^{*}\right)$

c) $v\left(s_{1}\left(t^{*}\right), t^{*}\right)=u_{2}\left(s_{1}\left(t^{*}\right), t^{*}\right)=u_{2}\left(s_{2}\left(t^{*}\right), t^{*}\right)=0$. 
Moreover, $u_{2}(0, t)>u_{1}(0, t)$ for $t<t^{*}$. Then

$$
v(0, t)>0, \quad t<t^{*}
$$

and

$$
v\left(s_{1}(t), t\right)=u_{2}\left(s_{1}(t), t\right)>0 .
$$

Since $v$ has the minimum value zero at $\left(s_{1}\left(t^{*}\right), t^{*}\right)$, the minimum principle to $v$ in $D_{t^{*}}^{1}$, we get $v_{x}\left(s_{1}\left(t^{*}\right), t^{*}\right)<0$, which is a contradiction by (a) to

$$
v_{x}\left(s_{1}\left(t^{*}\right), t^{*}\right)=u_{2 x}\left(s_{1}\left(t^{*}\right), t^{*}\right)=u_{2 x}\left(s_{2}\left(t^{*}\right), t^{*}\right)=0 .
$$

Then $t^{*} \neq t^{* *}$.

Claim 2: $t^{*}<t^{* *}$ is impossible.

On $\left[0, t^{*}\right], s_{1}(t)<s_{2}(t)$, whence $v\left(s_{1}(t), t\right)>0$. By definition, $v(0, t)>0$ for $t<t^{*}$ and $v\left(0, t^{*}\right)=0$. That implies $v\left(0, t^{*}\right)$ is a minimum value up to time $t^{*}$, whence $v_{x}\left(0, t^{*}\right)>0$, which contradicts

$$
v_{x}\left(0, t^{*}\right)=\beta\left[v\left(0, t^{*}\right)+\left(\left\|G_{2}\right\|_{1, t^{*}}-\left\|G_{1}\right\|_{1 . t^{*}}\right)\right]=\beta\left[\left\|G_{2}\right\|_{1 . t^{*}}-\left\|G_{1}\right\|_{1 . t^{*}}\right]<0 .
$$

Claim 3: $t^{* *}<t^{*}$ is impossible since:

Let $t^{* *}<t^{*}$. Since $v(0, t)>0, v\left(s_{1}(t), t\right)=u_{2}\left(s_{1}(t), t\right)>0$, for $t<t^{* *}$, the point $\left(s_{1}\left(t^{* *}\right), t^{* *}\right)$ is a minimum point for $v$ because $v\left(s_{1}\left(t^{* *}\right), t^{* *}\right)=u_{2}\left(s_{1}\left(t^{* *}\right), t^{* *}\right)=$ $u_{1}\left(s_{1}\left(t^{* *}\right), t^{* *}\right)=0$.

By the corner minimum principle,

$$
v_{x}\left(s_{1}\left(t^{* *}\right), t^{* *}\right)<0
$$

which contradicts

$$
v_{x}\left(s_{1}\left(t^{* *}\right), t^{* *}\right)=u_{2 x}\left(s_{2}\left(t^{* *}\right), t^{* *}\right)=0 .
$$

Thus the proposition is proved.

Acknowledgments. This paper has been partially sponsored by the Project 221 (CONICET-Rosario-Argentina) and the Project 2882/93 (CONICOR-CórdobaArgentina).

\section{REFERENCES}

[1] A. Fasano and M. Primicerio, General free-boundary problems for the heat equation. J. Math. Anal. Appl. I: II: 58, 202 2:31 (1977), 57, 694-723 (1977)

[2] A. Fasano and M. Primicerio, New results on parabolic free-boundary problems, Quart. Appl. Math. 38, 439-460 (1981)

[3] A. D. Solomon, V. Alexiades, and D. G. Wilson, The Stefan problem with a convective boundary condition, Quart. Appl. Math. 40, 203 217 (1982)

[4] E. Comparini, R. Ricci, and D. A. Tarzia, Remarks on a one dimensional Stefan problem related to the diffusion-consumption model, Z. Angew. Math. Mech. 64, 543550 (1984)

[5] J. R. Cannon and C. D. Hill, Remarks on a Stefan problem, J. Math. Mech. 17, 433-441 (1967)

[6] A. Friedman, Partial Differential Equations of Parabolic: Type, Prentice Hall, Englewood Cliffs, NJ, 1964 